\title{
Sobre el control central de la respiración: A propósito de una mujer con apnea obstructiva del sueño, enfermedad de Lyme y consumo crónico de opioides
}

\author{
César Manuel Salazar-Peña,* Martha Torres-Fraga, ${ }^{*}$ Jorge Martín Schalch-Ponce de León,** \\ Margarita Reyes-Zúñiga, ${ }^{*}$ Armando Castorena-Maldonado, ${ }^{*}$ Patricia Volkow-Fernández, ${ }^{\S}$ \\ José Rogelio Pérez-Padilla, José Luis Carrillo-Alduenda* ${ }^{*}$ \\ *Hospital Regional PEMEX Salamanca; Instituto Nacional de Enfermedades Respiratorias Ismael Cosío Villegas; \\ \$nstituto Nacional de Cancerología. \\ Trabajo recibido: 11-VIII-2015; aceptado: 19-II-2016
}

\begin{abstract}
RESUMEN. Existen diferentes trastornos que afectan el control central de la respiración y se manifiestan durante el dormir como apneas centrales. Aunque se conoce mucho de la fisiopatología y etiopatogenia de los diferentes síndromes de apnea central, aún no se han logrado develar todas sus características; asimismo, no se conoce la importancia de las neuroinfecciones en la disrupción de los mecanismos normales del control central de la respiración durante la noche. A continuación se presenta el caso de una mujer con afección neurológica por enfermedad de Lyme y uso crónico de opioides que causó modificaciones en la homeostasis respiratoria durante el sueño y que se resolvieron con uso de un dispositivo de presión positiva continua en la vía aérea.
\end{abstract}

Palabras clave: Enfermedad de Lyme, apnea obstructiva del sueño, apnea central del sueño, opioides.

ABSTRACT. There are several disorders that affect the central control of breathing during sleep and cause central apneas. Although much of the pathophysiology and pathogenesis of the different syndromes of central sleep apnea are known, their entire features persist unidentified; likewise, it is unknown the importance of the central nervous system infections in the disruption of the normal mechanisms of central control of breathing during sleep. We present the case of a woman with Neuro-Lyme disease and chronic use of opioids that caused changes in respiratory homeostasis during sleep and that resolved with the use of a continuous positive airway pressure device.

Key words: Lyme disease, obstructive sleep apnea, central sleep apnea, opioids.

\section{INTRODUCCIÓN}

La Academia Americana de Medicina de Sueño (AASM, por sus siglas en inglés) define a una apnea central (en el adulto) como una disminución del flujo respiratorio $\geq$ $90 \%$ con respecto a la respiración basal de al menos 10 segundos de duración y sin esfuerzo respiratorio. ${ }^{1}$ Este tipo de evento es la manifestación principal de diferentes síndromes que de acuerdo con la tercera Clasificación Internacional de los Trastornos de Sueño, en el adulto se catalogan de la siguiente manera: a) apnea central del sueño con respiración Cheyne-Stokes; b) apnea central del sueño debido a una condición médica sin respiración Cheyne-Stokes; c) apnea central del sueño debido a respiración periódica por altitud; d) apnea central del sueño debido a fármacos o sustancias (secundaria a opioides/narcóticos); e) apnea central del sueño primaria y f) apnea central del sueño emergente durante el tratamiento con un dispositivo de presión positiva (anteriormente denominadas apneas complejas). ${ }^{2}$

Estos síndromes son consecuencia de dos mecanismos fisiopatológicos (no excluyentes y frecuentemente asociados) en el control central de la respiración que se manifiestan exclusivamente durante el dormir: a) una mejor excreción de $\mathrm{CO}_{2}$ y ventilación más allá del límite del umbral apneico y b) un loop-gain o ganancia de asa alto. Vide Infra.

La enfermedad de Lyme (EL) es una infección ocasionada por Borrelia burgdorferi, sus manifestaciones son diversas y puede ocasionar problemas neurológicos, musculoesqueléticos, cardíacos y dermatológicos; ${ }^{3}$ además se ha asociado a alteraciones del dormir. 
Aunque se sabe mucho de la fisiopatología y etiopatogenia de los diferentes síndromes de apnea central (AC), aún no se han logrado develar todas sus características.

A continuación se presenta el caso de una mujer con afección neurológica por EL, uso crónico de opioides y apnea obstructiva del sueño, la interacción de estos factores causó modificaciones en la homeostasis respiratoria durante el sueño provocando apneas centrales durante el sueño que se resolvieron con el uso de un dispositivo de presión positiva continua en la vía aérea.

\section{CASO CLÍNICO}

Mujer de 41 años con EL diagnosticada por Western blot, referida por médico infectólogo con amplia experiencia en este padecimiento. Fue tratada con ceftriaxona y utilizaba tramadol por dolor crónico de extremidades, debido a afección neurológica se solicitó gammagrama cerebral con técnica SPECT/CT donde se documentó hipoperfusión generalizada y difusa en lóbulos frontal, temporal y parietal, pero con perfusión occipital conservada (figura 1A). Los síntomas de sueño fueron insomnio inicial, mala calidad de sueño, despertares frecuentes, ronquido habitual, cefalea matutina y somnolencia excesiva diurna. Tenía un índice de masa corporal de 32.5 $\mathrm{kg} / \mathrm{m}^{2}$, circunferencia de cuello de $40 \mathrm{~cm}$ y $\mathrm{SpO}_{2}$ en vigilia $94 \%$. En una primera polisomnografía (PSG) (tabla 1 y figura 2) se demostró apnea obstructiva del sueño grave. Durante la titulación de un dispositivo de presión positiva continua en la vía aérea (CPAP) se observaron apneas centrales con un patrón de respiración periódica a un umbral de $7 \mathrm{cmH}_{2} \mathrm{O}$ (figuras 2 y 3 ), estos eventos persistieron a pesar de retirar la ventilación mecánica (figura 2). Se prescribió CPAP a $6 \mathrm{cmH}_{2} \mathrm{O}$ por 30 días y se suspendió el tramadol. En una segunda PSG de titulación no se observaron apneas centrales y $6 \mathrm{cmH}_{2} \mathrm{O}$ de CPAP fueron suficientes para eliminar todos los eventos obstructivos (tabla 1). En el gammagrama cerebral de control (figura 1B), 30 días posteriores al uso continuo de CPAP se observó perfusión cerebral normal. Al presente, se encuentra sin somnolencia y con disminución de la fatiga y del dolor.

\section{DISCUSIÓN}

Hay dos conceptos que tienen que revisarse para entender la génesis de la AC, éstos son el umbral apneico y la ganancia de asa.

Durante la vigilia, un aumento transitorio del impulso central de la respiración y la consiguiente hiperventi-
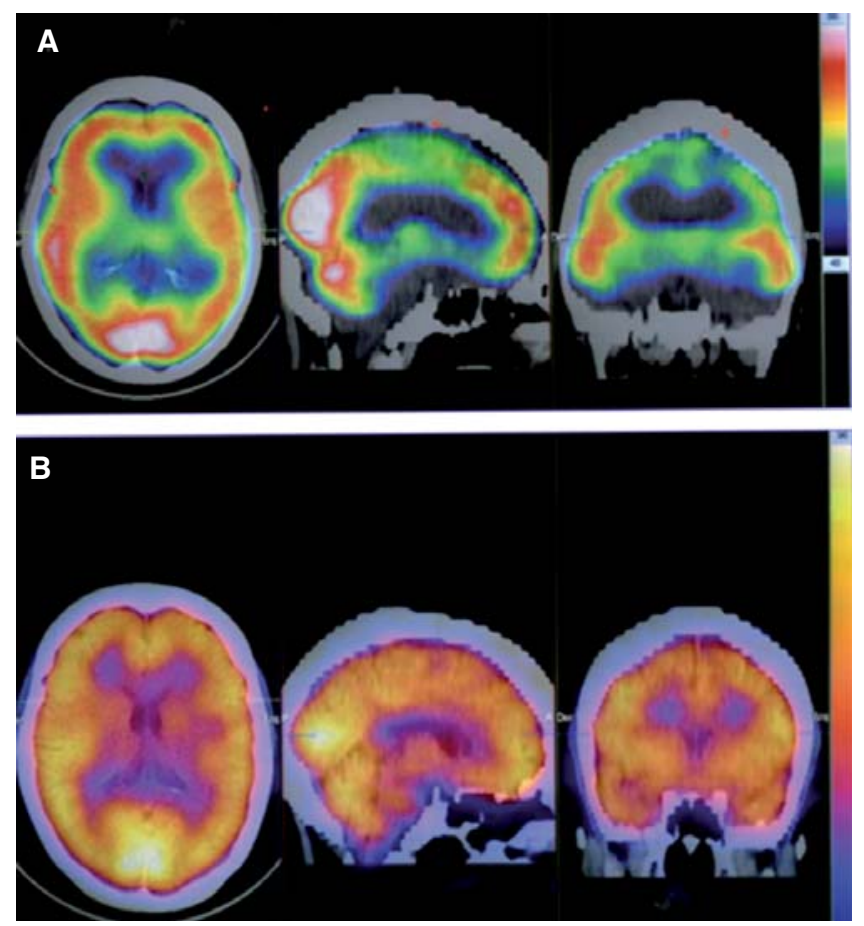

Figura 1. Cortes axial, sagital y coronal de fusión SPECT/ CT de gammagrafía de perfusión cerebral con ${ }^{99 m} T c-E C D$. A) Estudio basal donde se aprecia hipoperfusión difusa y bilateral en lóbulos frontal, temporal y con mayor afectación en el parietal. Es de resaltar que la perfusión en el lóbulo occipital está conservada. B) Estudio para valorar la respuesta al tratamiento posterior a CPAP, en donde se observa resolución completa de los defectos de perfusión cerebral (estudio normal).

lación no ocasiona apneas, ya que éste va seguido de un cese del estímulo ventilatorio y la respiración vuelve paulatinamente a su basal. Durante el sueño, este sistema de estabilización de la respiración se ve sobrepasado, pues se pierde el control volitivo y la respiración es controlada de manera autónoma por quimiorreceptores, siendo el principal estímulo el nivel de $\mathrm{CO}_{2}$ en plasma. ${ }^{4,5} \mathrm{El}$ umbral apneico se define como el nivel de $\mathrm{CO}_{2}$ en plasma por abajo del cual se presenta una apnea central. Los despertares transitorios del sueño o alertamientos son impulsos adicionales que aumentan la frecuencia respiratoria, reducen la resistencia de vía aérea superior y permiten la hiperventilación, manifestación común durante la transición de la vigilia a las primeras etapas de sueño. Como mecanismo protector existe una hipoventilación «fisiológica» relacionada al colapso de la vía aérea superior y a la caída del volumen minuto por disminución en el volumen corriente sin incremento en la frecuencia respiratoria. ${ }^{6-8}$

Los sitios anatómicos relacionados con la AC y la inestabilidad del sistema respiratorio durante el sueño 
Tabla 1. Arquitectura de sueño e índices respiratorios de la primera y segunda PSG. Se aprecia un IAH grave en la parte basal de la primera PSG que se incrementa en la fase de titulación de CPAP a expensas de apneas centrales. En la segunda PSG tanto los eventos centrales como los obstructivos fueron controlados.

\begin{tabular}{|c|c|c|c|}
\hline & Primera PSG, parte basal & Primera PSG, CPAP & Segunda PSG \\
\hline Eficiencia \% & 81 & 83 & 81 \\
\hline N1 (\%TST) & 6.1 & 13 & 6.1 \\
\hline N2 (\%TST) & 57.6 & 45 & 57.6 \\
\hline N3 (\%TST) & 12.3 & 22 & 12.3 \\
\hline $\mathrm{R}(\% \mathrm{TST})$ & 24.1 & 20 & 24.1 \\
\hline $\mathrm{IA} \mathrm{h}^{-1}$ & 4.6 & 6.2 & 4.9 \\
\hline IAR h-1 & 0.3 & 1.1 & 0.3 \\
\hline$I A C h^{-1}$ & 1.6 & 104 & 0.1 \\
\hline $\mathrm{IAO} \mathrm{h}^{-1}$ & 1.8 & 0 & 0.1 \\
\hline $\mathrm{IAM} \mathrm{h}^{-1}$ & 1 & 0 & 0 \\
\hline $\mathrm{IH} \mathrm{h}^{-1}$ & 25.9 & 9.3 & 3 \\
\hline $\mathrm{IAH} \mathrm{h-1}$ & 38.6 & 120 & 3.3 \\
\hline $\mathrm{SpO}_{2}$ promedio $\%$ & 92 & 87 & 92 \\
\hline
\end{tabular}

Abreviaturas: CPAP = presión positiva continua en la vía aérea; $\mathrm{h}^{-1}=$ eventos por hora de sueño; $I A=$ índice de alertamientos; $I A C=$ índice de apneas centrales; IAH = índice de apnea hipopnea; IAM = índice de apneas mixtas; IAO = índice de apneas obstructivas; IAR = índice de alertamientos respiratorios; $\mathrm{IH}$ = índice de hipopneas; $\mathrm{PSG}=$ polisomnografía; $\mathrm{SpO}_{2}=$ saturación por pulsoximetría; TST = tiempo total de sueño.

se han investigado en modelos murinos, la evidencia apunta a un complejo circuito neuronal que incluye a los cuerpos carotídeos y sus proyecciones aferentes, los quimiorreceptores del núcleo del tracto solitario (NTS), médula ventral lateral (VLM) y a las neuronas quimiosensibles al $\mathrm{CO}_{2}$ del núcleo retrotrapezoideo (RTN). ${ }^{9-11}$

Aunque el umbral de la apnea central hipocápnica es muy sensible durante el sueño de no movimientos oculares rápidos (noMOR) sobre todo al inicio del sueño, no hay un umbral aparente durante el sueño de movimientos oculares rápidos (MOR), especialmente en su componente fásico, incluso con marcadas reducciones en la $\mathrm{PaCO}_{2}$; así, las apneas centrales y la respiración periódica que acompaña a la insuficiencia cardíaca con respiración Cheyne-Stokes o las que se presentan durante la hipoxia de las grandes altitudes rara vez se presentan en este estadío de sueño. ${ }^{12,13}$ Se ha postulado que de manera análoga al estado de vigilia, los incrementos irregulares y esporádicos del impulso inspiratorio central durante el sueño MOR anulan la inhibición respiratoria causada por la hipocapnia. ${ }^{14}$

La ganancia de asa es un término utilizado en ingeniería que define la ganancia de un sistema de retroalimentación negativo. En este caso el sistema control es el que regula la ventilación, el cual responde y retroalimenta de manera negativa en respuesta a una perturbación que lo inestabilice. ${ }^{15}$ La ganancia de asa se define matemáticamente como una razón entre la respuesta correctiva del sistema (p. ej. taquipnea o hiperpnea) y la magnitud de la perturbación ( $p$. ej. apnea). ${ }^{16}$ La aparición de las apneas centrales y respiración periódica relacionadas con episodios de hiperventilación durante el sueño varían mucho en función de la ganancia del sistema de control de las vías respiratorias, quienes tienen una elevada ganancia de asa están predispuestos a la inestabilidad del sistema respiratorio, en otras palabras, predispuestos a la AC y a la respiración periódica; al contrario, aquéllos con una baja ganancia de asa son resistentes a la inestabilidad del sistema y por consiguiente resistentes a la respiración periódica y AC. Existen diferentes componentes dentro del sistema de control de ganancia de asa: la ganancia control (controller gain), la ganancia base (plant gain) y la ganancia mixta (mixing gain). La primera queda representada en las regiones anatómicas responsables del control central de la respiración, la ganancia base se refiere a la eficacia en la excreción del $\mathrm{CO}_{2}$ y la ganancia mixta al retraso impuesto por la unión del $\mathrm{CO}_{2}$ a la hemoglobina y al tiempo de retraso de la señal o tiempo de circulación. ${ }^{17,18}$

De los distintos síndromes de AC, dos tipos en específico tienen que ser considerados en este caso clínico: AC emergente durante el tratamiento con un dispositivo de PP (apneas complejas) y la AC debida a fármacos.

Hasta el momento existe controversia respecto a la naturaleza de la $A C$ del sueño emergente durante el tratamiento como una enfermedad. ${ }^{16,19}$ Dependiendo de la fuente consultada se reduce a la presencia de $A C$ en pacientes con síndrome de apnea obstructiva del sueño (SAOS) en el inicio de su tratamiento con CPAP. ${ }^{2}$ Se presenta aproximadamente en $10 \%$ de todas las titulaciones con CPAP, desapareciendo con la evo- 


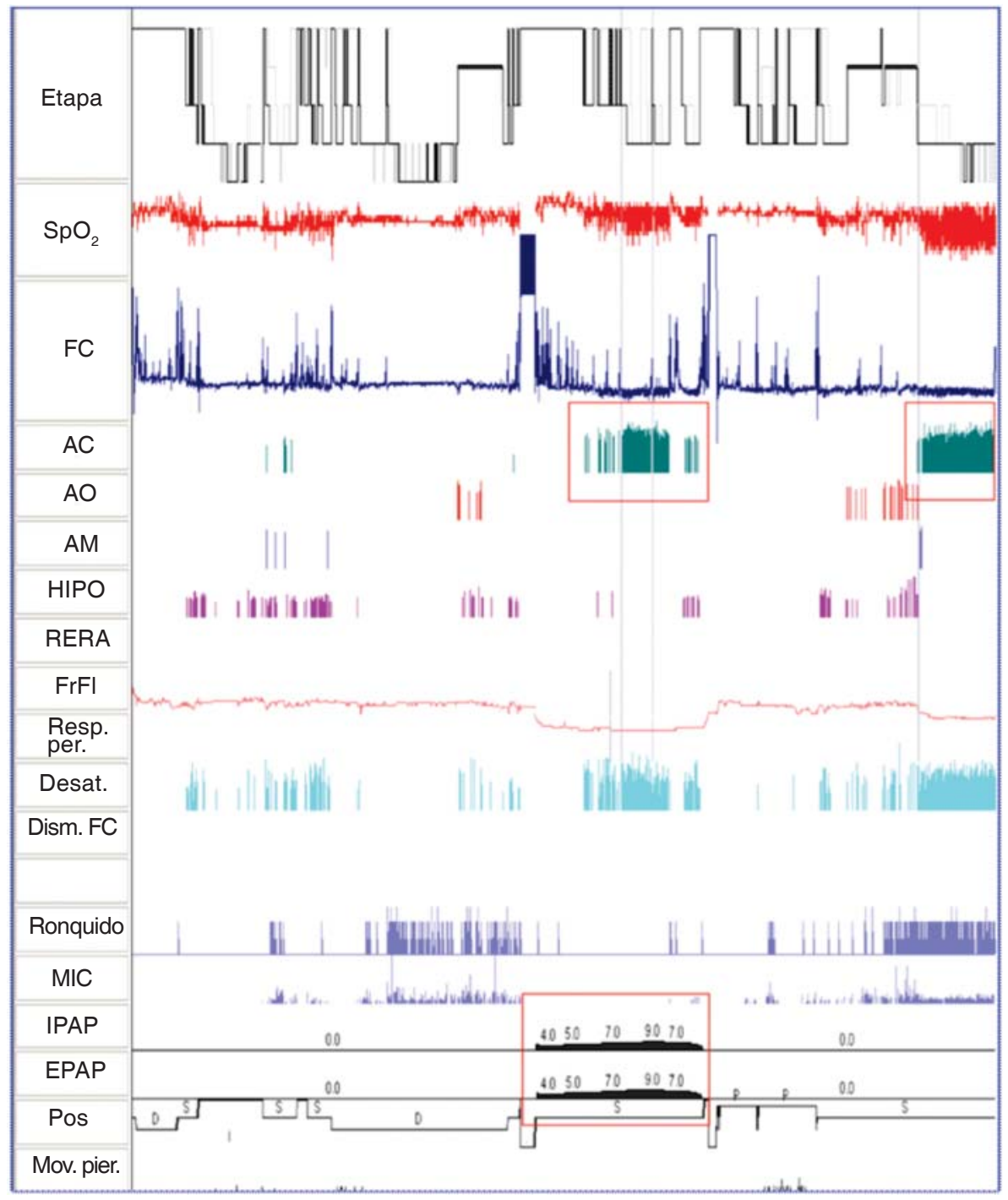

Figura 2.

Resumen de la primera PSG, en cuadro rojo se señalan los episodios de respiración periódica y apneas centrales; el primero coincide con el uso de CPAP y el segundo se presenta sin equipo de presión positiva en la vía aérea.

Imagen a color en: www.medigraphic. com/neumologia

Abreviaturas: $\mathrm{AC}=$ apneas centrales; $\mathrm{AM}$ = apneas mixtas; $A O=$ apneas obstructivas; Desat = desaturaciones; EPAP = presión espiratoria; HIPO = hipopneas; IPAP = presión inspiratoria; $\mathrm{SpO}_{2}=$ saturación por pulsoximetría. lución del tratamiento; ${ }^{20,21}$ son pacientes en su mayoría del sexo masculino ${ }^{22,23}$ que tienen predominantemente apneas mixtas $\mathrm{u}$ obstructivas durante la $\mathrm{PSG} .{ }^{16} \mathrm{La}$ teoría más aceptada acerca de la génesis de este tipo de apneas es el aumento en la eficacia para excretar el $\mathrm{CO}_{2}$ al disminuir la resistencia de la vía aérea superior gracias al CPAP, logrando sobrepasar el límite del umbral apneico, generándose una apnea central.2,24 Existen otras hipótesis aún menos estudiadas como la sobretitulación con CPAP relacionada con la activación de receptores de estiramiento pulmonar que inhiben la emisión central del impulso motor respiratorio; otra teoría es la excreción de $\mathrm{CO}_{2}$ del espacio muerto anatómico a través de fuga por la mascarilla o la boca debido a altas presiones del CPAP. El iniciar el tratamiento con CPAP con una presión inadecuada podría empeorar en algunos casos la calidad del sueño y un aumento en las transiciones de vigilia a sueño contribuiría a aumentar las apneas centrales de transición. Por otro lado, el uso de dispositivos de dos presiones o binivel, en caso de que estuviera indicado, ${ }^{25}$ podría contribuir a la presencia de apneas centrales al aumentar el volumen corriente llevando también al $\mathrm{CO}_{2}$ por debajo del umbral apneico. ${ }^{19}$ Aproximadamente $2 \%$ de las apneas complejas no se resuelven con el tiempo y no existe un tratamiento bien establecido para las mismas, se han utilizado diversas opciones como el uso de hipnóticos, acetazolamida y modos ventilatorios más avanzados al CPAP convencional como la servoventilación adaptativa y la ventilación modulada anticíclica. ${ }^{24,26}$ No debe confundirse con la apnea del sueño refractaria al uso de CPAP, esta última se presenta por una inadecuada presión de CPAP por falta de apego al dispositivo de presión positiva, por presencia de apneas residuales, 


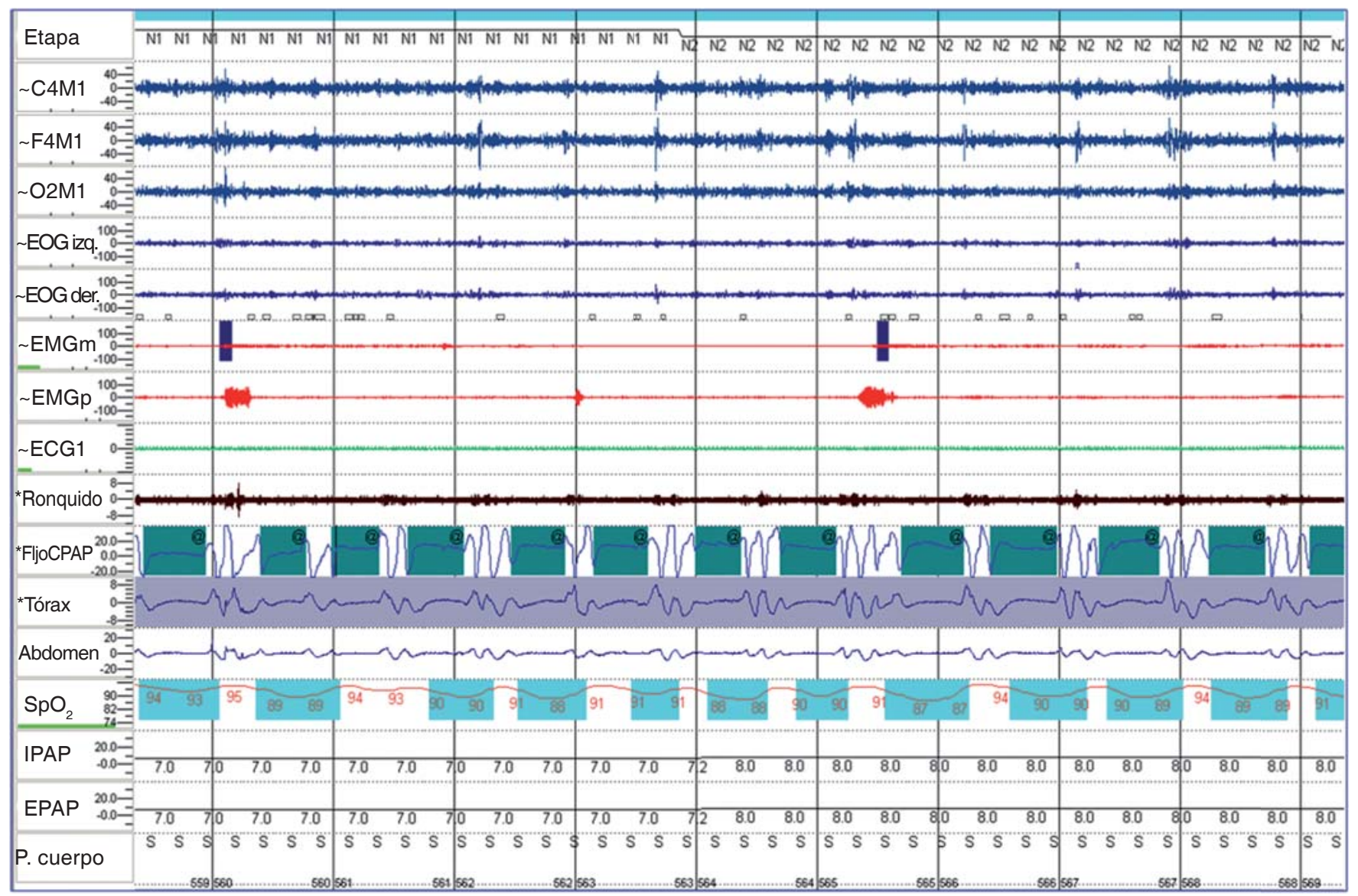

Figura 3. Fragmento de 5 minutos de la primera PSG durante la titulación de CPAP, en cuadros verdes están señaladas las apneas centrales que tienen forma periódica.

Imagen a color en: www.medigraphic.com/neumologia

por fuga en la mascarilla o por otros trastornos del sueño subyacente o distinto a la apnea del sueño. ${ }^{2,19}$

Las AC inducidas por el consumo de fármacos o sustancias se producen en pacientes con ingesta crónica de opioides, debido principalmente a dolor crónico o a consumo de drogas ilícitas. El uso de narcóticos para el dolor ha aumentado en frecuencia ${ }^{27}$ en ciertas situaciones abusando de los mismos, siendo la AC inducida por narcóticos una complicación emergente que apenas está comenzando a tener impacto en los círculos médicos. ${ }^{28}$ Ciertos tipos de receptores de opioides están ligados a la respiración de manera independiente, los receptores $\mathrm{Mu}$ en el tronco encefálico producen inhibición de distintos parámetros respiratorios, así como los receptores Delta en el NTS. En el sistema nervioso central en la superficie ventral de la médula y el puente existen sitios anatómicos importantes relacionados con la inhibición de la respiración inducida por opioides entre los que se encuentran los receptores Kappa. ${ }^{29}$ Las AC inducidas por narcóticos tienen una prevalencia aproximada de entre 10 y $50 \%$. Clínicamente se caracterizan por bradipnea y en algunos casos las apneas son dosis dependientes con períodos largos de hipoventilación, hipoxemia importante y respiración periódica, situación que mejora durante el sueño REM; asimismo, pueden experimentar remisión de su cuadro clínico con la reducción y suspensión de la dosis de narcóticos como en el caso de nuestra paciente. ${ }^{2,28}$ El tratamiento de esta entidad no está bien estudiado. Se han empleado analgésicos locales o analgésicos no-narcóticos para reducir la dosis del narcótico utilizado. ${ }^{2}$ También se ha propuesto a la servoventilación adaptativa como solución a este tipo de eventos. ${ }^{30}$

La EL ocasiona varias alteraciones de sueño como insomnio inicial y de mantenimiento, disminución de la eficiencia de sueño, sueño no reparador y de mala calidad y somnolencia excesiva diurna; ${ }^{31}$ sin embargo, una posible asociación con trastornos respiratorios del dormir no ha sido descrita. 
Presentamos el caso de una mujer con afección a sistema nervioso de EL, consumo crónico de tramadol, hipoperfusión cerebral y apnea obstructiva del sueño grave; quien durante la titulación de CPAP desarrolló apneas centrales con patrón de respiración periódica. La perfusión cerebral mejoró después de 30 días de tratamiento con CPAP y las apneas centrales desaparecieron al retirar el opioide. Así, podemos inferir que la mejoría en la perfusión cerebral fue debido al tratamiento de las apneas obstructivas, mientras que las apneas centrales fueron secundarias al consumo de narcóticos. Desafortunadamente, la contribución de la afección neurológica por la EL en el desarrollo de estas apneas centrales persiste como una interrogante.

\section{CONCLUSIÓN}

El caso ilustra las complejas interacciones que existen entre el control central respiratorio y la colapsabilidad de la vía aérea superior y cómo éstas pueden verse modificadas por diversos factores externos como una infección, medicamentos y trastornos respiratorios del sueño.

\section{REFERENCIAS}

1. Berry RB, Brooks R, Garnaldo CE, Harding SM. Marcus $\mathrm{CL}$ and Vaughn BU for the American Academy of Sleep Medicine. The AASM Manual for the Scoring of Sleep and Associated Events, Rules, Terminology and Technical Specifications. Version 2.3. www.aasmnet.org, Darien, Illinois: American Academy of Sleep Medicine; 2012.

2. American Academy of Sleep Medicine. International Classification of Sleep Disorders. 3rd ed. Darien II: American Academy of Sleep Medicine; 2014.

3. Marques A. Chronic Lyme disease: a review. Infect Dis Clin North Am 2008;22(2):341-360, vii-viii. doi: 10.1016/j. idc.2007.12.011.

4. Badr MS, Skatrud JB, Dempsey JA. Determinants of poststimulus potentiation in humans during NREM sleep. J Appl Physiol (1985) 1992;73(5):1958-1971.

5. Eldridge FL. Central neural respiratory stimulatory effect of active respiration. J Appl Physiol 1974;37(5):723-735.

6. Horner RL, Sanford LD, Pack Al, Morrison AR. Activation of a distinct arousal state immediately after spontaneous awakening from sleep. Brain Res 1997;778(1):127-134.

7. Xie A, Wong B, Phillipson EA, Slutsky AS, Bradley TD. Interaction of hyperventilation and arousal in the pathogenesis of idiopathic central sleep apnea. Am J Respir Crit Care Med 1994;150(2):489-495.

8. Younes M. Role of arousals in the pathogenesis of obstructive sleep apnea. Am J Respir Crit Care Med 2004;169(5):623-633.

9. Dauger S, Pattyn A, Lofaso F, et al. Phox $2 b$ controls the development of peripheral chemoreceptors and affer- ent visceral pathways. Development 2003;130(26):66356642.

10. Takakura AC, Moreira TS, Colombari E, West GH, Stornetta RL, Guyenet PG. Peripheral chemoreceptor inputs to retrotrapezoid nucleus (RTN) $\mathrm{CO}_{2}$-sensitive neurons in rats. J Physiol 2006;572(Pt 2):503-523.

11. Stornetta RL, Moreira TS, Takakura AC, et al. Expression of Phox $2 b$ by brainstem neurons involved in chemosensory integration in the adult rat. J Neurosci 2006;26(40):10305-10314.

12. Dempsey JA, Veasey SC, Morgan BJ, O'Donnell CP. Pathophysiology of sleep apnea. Physiol Rev 2010;90(1):47112. doi: 10.1152/physrev.00043.2008.

13. Hanly PJ, Millar TW, Steljes DG, Baert R, Frais MA, Kryger $\mathrm{MH}$. Respiration and abnormal sleep in patients with congestive heart failure. Chest 1989;96(3):480-488.

14. Orem J. Central respiratory activity in rapid eye movement sleep: augmenting and late inspiratory cells. Sleep 1994;17(8):665-673.

15. Khoo MC, Kronauer RE, Strohl KP, Slutsky AS. Factors inducing periodic breathing in humans: a general model. J Appl Physiol Respir Environ Exerc Physiol 1982;53(3):644-659.

16. Gay PC. Complex sleep apnea: it really is a disease. J Clin Sleep Med 2008;4(5):403-405.

17. Cherniack NS, Longobardo GS. Mathematical models of periodic breathing and their usefulness in understanding cardiovascular and respiratory disorders. Exp Physiol 2006;91(2):295-305.

18. Khoo MC. Determinants of ventilatory instability and variability. Respir Physiol 2000;122(2-3):167-182.

19. Malhotra A, Bertisch S, Wellman A. Complex sleep apnea: it isn't really a disease. J Clin Sleep Med 2008;4(5):406-408.

20. Dernaika T, Tawk M, Nazir S, Younis W, Kinasewitz GT. The significance and outcome of continuous positive airway pressure related central sleep apnea during split-night sleep studies. Chest 2007;132(1):81-87.

21. Javaheri S, Smith J, Chung E. The prevalence and natural history of complex sleep apnea. J Clin Sleep Med 2009;5(3):205-211.

22. Morgenthaler TI, Kagramanov V, Hanak V, Decker PA. Complex sleep apnea syndrome: is it a unique clinical syndrome? Sleep 2006;29(9):1203-1209.

23. Lehman S, Antic NA, Thompson C, Catcheside PG, Mercer J, McEvoy RD. Central sleep apnea on commencement of continuous positive airway pressure in patients with a primary diagnosis of obstructive sleep apnea-hypopnea. J Clin Sleep Med 2007;3(5):462-466.

24. White DP. Central sleep apnea. Med Clin North Am 1985;69(6):1205-1219.

25. Kushida CA, Chediak A, Berry RB, et al.; Positive Airway Pressure Titration Task Force; American Academy of Sleep Medicine. Clinical guidelines for the manual titration of positive airway pressure in patients with obstructive sleep apnea. J Clin Sleep Med 2008;4(2):157171.

26. White DP, Zwillich CW, Pickett CK, Douglas NJ, Findley LJ, Weil JV. Central sleep apnea. Improvement with acet- 
azolamide therapy. Arch Intern Med 1982;142(10):18161819.

27. Elliott AM, Smith BH, Penny KI, Smith WC, Chambers WA. The epidemiology of chronic pain in the community. Lancet 1999;354(9186):1248-1252.

28. Eckert DJ, Jordan AS, Merchia P, Malhotra A. Central sleep apnea: pathophysiology and treatment. Chest 2007;131(2):595-607.

29. Shook JE, Watkins WD, Camporesi EM. Differential roles of opioid receptors in respiration, respiratory disease, and opiate-induced respiratory depression. Am Rev Respir Dis 1990;142(4):895-909.

30. Javaheri S, Malik A, Smith J, Chung E. Adaptive pressure support servoventilation: a novel treatment for sleep apnea associated with use of opioids. J Clin Sleep Med 2008;4(4):305-310.
31. Greenberg HE, Ney G, Scharf SM, Ravdin L, Hilton E. Sleep quality in Lyme disease. Sleep 1995;18(10):912916.

\section{$\triangle$ Correspondencia:}

Dr. José Luis Carrillo Alduenda

Instituto Nacional de Enfermedades Respiratorias Ismael Cosío Villegas.

Calzada de Tlalpan Núm. 4502,

Colonia Sección XVI, C.P. 14080,

Delegación Tlalpan, Ciudad de México.

Correo electrónico: jlcarrillo14@ hotmail.com

Los autores declaran no tener conflicto de intereses. 